\title{
Serial exercise testing in children, adolescents and young adults with Senning repair for transposition of the great arteries
}

\author{
Roselien Buys ${ }^{1 *}$, Werner Budts ${ }^{2}$, Tony Reybrouck ${ }^{1,3}$, Marc Gewillig ${ }^{4}$ and Luc Vanhees ${ }^{1}$
}

\begin{abstract}
Background: Patients with Senning repair for complete transposition of the great arteries (d-TGA) show an impaired exercise tolerance. Our aim was to investigate changes in exercise capacity in children, adolescents and adults with Senning operation.

Methods: Peak oxygen uptake (peak $\mathrm{VO}_{2}$ ), oxygen pulse and heart rate were assessed by cardiopulmonary exercise tests (CPET) and compared to normal values. Rates of change were calculated by linear regression analysis. Right ventricular (RV) function was assessed by echocardiography.

Results: Thirty-four patients (22 male) performed 3.5 (range 3-6) CPET with an interval of $\geq 6$ months. Mean age at first assessment was $16.4 \pm 4.27$ years. Follow-up period averaged $6.8 \pm 2$ years. Exercise capacity was reduced $(p<0.0005)$ and the decline of peak $\mathrm{VO}_{2}(-1.3 \pm 3.7 \% / y e a r ; p=0.015)$ and peak oxygen pulse $(-1.4 \pm 3.0 \%$ /year; $p=0.011$ ) was larger than normal, especially before adulthood and in female patients ( $p<0.01)$. During adulthood, RV contractility changes were significantly correlated with the decline of peak oxygen pulse $(r=-0.504 ; p=0.047)$.

Conclusions: In patients with Senning operation for d-TGA, peak $\mathrm{VO}_{2}$ and peak oxygen pulse decrease faster with age compared to healthy controls. This decline is most obvious during childhood and adolescence, and suggests the inability to increase stroke volume to the same extent as healthy peers during growth. Peak $\mathrm{VO}_{2}$ and peak oxygen pulse remain relatively stable during early adulthood. However, when RV contractility decreases, a faster decline in peak oxygen pulse is observed.
\end{abstract}

Keywords: Exercise capacity, Transposition of the great arteries, Senning repair, Median term follow-up

\section{Background}

Until about 1980, most children with complete transposition of the great arteries (d-TGA) underwent the Mustard or the Senning operation. These atrial switch techniques were replaced when an arterial switch procedure was introduced which allows for a systemic left ventricular function. However, $75 \%$ to $80 \%$ of the patients with Mustard or Senning repair have survived into adulthood and are now regularly visiting the outpatients clinics for grown-up congenital heart disease $[1,2]$.

It has often been shown that children, adolescents and adults with Senning repair for d-TGA have a

\footnotetext{
*Correspondence: roselien.buys@faber.kuleuven.be

${ }^{1}$ Department of Rehabilitation Sciences, Research Center for Cardiovascular and Respiratory Rehabilitation, KULeuven, Tervuursevest 101, Leuven 3001, Belgium

Full list of author information is available at the end of the article
}

substantially reduced exercise capacity, which probably lowers faster with age compared to healthy controls [2-8]. This lower exercise capacity has been explained by chronotropic incompetence, impaired systemic ventricular function, impaired function of the intra-atrial conduit, the inability to increase stroke volume, impaired lung function with ventilation/perfusion mismatch and peripheral deconditioning $[2,6,7]$. Moreover, a poor exercise capacity identifies d-TGA patients at risk for hospitalization and death [9].

Cross-sectional studies show evidence for a general decrease in exercise capacity in patients with atrial switch repair for d-TGA $[4,6]$. Reybrouck et al. performed a longitudinal study in children and adolescents with atrial switch operation [3]. They concluded that in the overall group, the exercise performance remained

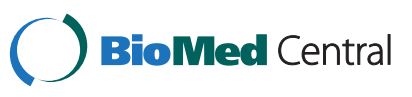


stable during a follow-up of $3.5 \pm 2$ years, but that in individual patients, a decreasing exercise capacity was correlated with the development of hemodynamic lesions [3].

At present, information on the evolution of the exercise capacity in adult patients with Senning operation performed during childhood is not available and possible differences in the evolution of the exercise capacity between children and adults have not been investigated yet.

Therefore the aim of our study was to investigate the change of the exercise capacity and the cardiovascular status in children, adolescents and young adults with Senning repair for transposition of the great arteries.

\section{Methods}

\section{Patients}

All patients with Senning repair for d-TGA who performed at least 3 cardiopulmonary exercise tests until exhaustion, at least 6 months apart from each other were included in the serial study. Their exercise tests were performed at the occasion of their follow-up visits to the outpatient clinic of paediatric cardiology or adult congenital heart disease of our hospital, therefore no further written informed consent was needed according to the regulations of the ethic committee. The study was approved by the Institutional Review board of the University Hospitals Leuven.

\section{Cardiopulmonary exercise tests}

Patients younger than 16 years old performed a graded maximal exercise test on the treadmill. The treadmill was set at a speed of $5,6 \mathrm{~km} / \mathrm{h}$. The gradient was increased every minute by $2 \%$ until exhaustion was reached. The children were encouraged to perform a true maximal effort. Oxygen uptake and carbon dioxide output were measured on a breath-by-breath basis by a computerised system with fast responding electronic gas analysers (Med Graphics, Ultima). Inspiratory and expiratory flow was measured with a Pitot flow meter. The system was calibrated before each exercise test with test gas of known composition. Heart rhythm was continuously monitored during exercise and a twelve-lead electrocardiogram was recorded every minute. Patients' results were compared with reference values and expressed as percentage of normal. The normal values were obtained from a large cohort of healthy school children $(\mathrm{n}=234$, male/female, age range $5.7-18.5$ years $)$ who performed the same exercise testing protocol [10].

Patients from 16 years and older performed maximal exercise tests on a bicycle ergometer (Ergometrics 800S, Ergometrics, Bitz, Germany). The initial workload of $20 \mathrm{~W}$ was increased by $20 \mathrm{~W}$ every minute until exhaustion. Patients were encouraged to perform a true maximal effort. A twelve-lead electrocardiogram and respiratory data through breath-by-breath analysis were continuously registered with a computerized system (Oxygen AlphaR, Jaeger, Mijnhardt, Bunnik, The Netherlands). The gas analyzers and the flow meter were calibrated before each test according to the manufacturer's instructions. Oxygen uptake $\left(\mathrm{VO}_{2}\right)$ was determined from the continuous measurement of the oxygen concentration in the inspired and expired air. Results were compared to results of a control group of 368 men and 158 women (age range 16-65 years old) who performed the same exercise testing protocol in our laboratory, and expressed as percentage of normal values according to age, gender and weight.

Exercise performance was assessed by determination of the peak oxygen uptake (peak $\mathrm{VO}_{2}$ ), and peak heart rate (HR). Peak oxygen pulse was calculated by dividing peak $\mathrm{VO}_{2}$ expressed in $\mathrm{ml} / \mathrm{min} / \mathrm{kg}$ by peak $\mathrm{HR}$, which was then multiplied by ten in order to obtain values greater than 1.

\section{Echocardiography}

Routine transthoracic echocardiography was performed in all patients at all assessments with standard grayscale, and Doppler imaging examinations. All echocardiographic studies were performed with the patient in a supine position. As it is general practice, qualitative evaluations were used for the evaluation of right ventricular hypertrophy $(0=$ no hypertrophy, 1 =hypertrophy), right ventricular dilatation (no, slight, moderate and severe dilatation, scaling from 1 to 4), right ventricular contractility (normal, mild, moderate, and severe dysfunction, scaling from 1 to 4 ) and tricuspid regurgitation $(0-4 / 4)$.

\section{Statistical analysis}

SAS statistical software version 9.3 for windows (Sas Institute Inc, Cary, NC, USA) was used for the analysis. Data are reported as means and standard deviation or as numbers for dichotomous variables. Exercise capacity was compared with average (mean percent predicted value $=100 \%$ ) using a one-sample t-test. Mean rate of change was calculated for each patient by linear regression analysis. The one-sample $\mathrm{t}$-test was again used for comparison of the rate of change with average (mean percent predicted change $=0 \%$ ). Student's paired t-test, or paired sample Wilcoxon signed rank sum test when appropriate, was used to compare differences between measures from initial and final assessments. Pearson or Spearman correlation coefficients were calculated to assess possible relationships between rates of change in exercise measures and age at Senning operation or changes in echocardiographic parameters. All statistical tests were 2 -sided with a significance level of $\leq 0.05$. 


\section{Results}

\section{Patients}

A total group of 34 patients with Senning operation for d-TGA were included in our study. The characteristics of these patients are represented in Table 1 . Age at the initial evaluation ranged between 8 and 23 years and at the last assessment the age of the patients ranged between 13 and 29 years. Median time between the first and the last assessment was 6.6 years and ranged between 1.6 and 11 years. All patients were in New York Heart Association class I at all assessments.

\section{Exercise measures}

Exercise data from the first and the last assessment are shown in Table 2. For all assessments, percentages of predicted peak $\mathrm{VO}_{2}$, peak $\mathrm{HR}$ and peak oxygen pulse were significantly lower than the normal values.

As shown in Table 3, a significant decline from the initial to the final assessment was found for peak $\mathrm{VO}_{2}$ and peak oxygen pulse when expressed as percentages of predicted values $(\mathrm{p}=0.015$ and $\mathrm{p}=0.011$ respectively). When our patient group was divided into two groups based on the age at first assessment, this decrease was only significant in patients younger than 16 years old at first assessment $(\mathrm{p}<0.01)$. Further subanalysis by gender revealed a significant decline of peak $\mathrm{VO}_{2}$ (decline $-3.3 \pm 2.3 \%$ /year; $\mathrm{p}=0.026)$ and peak oxygen pulse (decline $-3.0 \pm 3.9 \% /$ year; $\mathrm{p}=0.022$ ) in female patients but not in male patients.

Individual changes in peak $\mathrm{VO}_{2}$ and peak oxygen pulse expressed as a percentage of the predicted normal value are presented in figure 1 . When a cut off point was set at 18 year, the change in peak $\mathrm{VO}_{2} \%$ and peak oxygen pulse was higher before $(\mathrm{p}<0.01)$ then after the cut off point.

Chronotropic incompetence, as defined as a peak HR lower than $80 \%$ of the normal value, was only present in 2 patients $(6 \%)$ at the initial assessment and in 3 patients (9\%) at the final assessment. The rate of change in peak HR was not significantly different from a normal population. Rates of change in peak $\mathrm{VO}_{2}$, peak $\mathrm{HR}$ and peak oxygen pulse were not related to age at surgical repair.

\section{Echocardiography}

Echocardiographic findings are summarized in Table 2. At the first assessment all patients had RV hypertrophy,

Table 1 Patient characteristics $(n=34)$

\begin{tabular}{ll}
\hline Male & $\mathbf{2 2 ( 6 6 )}$ \\
Age at repair (months) & $4(0.4-14)$ \\
Time between first and last assessment (years) & $6.8 \pm 2.0$ \\
Number of assessments per patient & $3.5(3-6)$ \\
\hline
\end{tabular}

Data are expressed as mean \pm standard deviation or as median (range).
Table 2 Comparison of initial with final assessment

\begin{tabular}{|c|c|c|c|}
\hline & First test & Last test & $p$ \\
\hline Age (years) & $16.4 \pm 4.27$ & $22.9 \pm 4.30$ & $<0.0001$ \\
\hline Height (cm) & $161 \pm 16$ & $171 \pm 11$ & 0.0002 \\
\hline Weight (kg) & $51.8 \pm 16.1$ & $65.8 \pm 11.5$ & $<0.0001$ \\
\hline Peak oxygen uptake $(\mathrm{ml} / \mathrm{min} / \mathrm{kg})$ & $33.6 \pm 6.65$ & $27.4 \pm 6.96$ & $<0.0001$ \\
\hline Peak oxygen uptake $(\%)^{\circ}$ & $73 \pm 12^{* *}$ & $65 \pm 13^{* *}$ & 0.002 \\
\hline Peak heart rate (beats/min) & $179 \pm 18$ & $174 \pm 15$ & 0.022 \\
\hline Peak heart rate $(\%)^{\circ}$ & $94 \pm 10^{*}$ & $94 \pm 8^{* *}$ & 0.69 \\
\hline Peak oxygen pulse (ml/kg/beat) & $19.0 \pm 4.39$ & $15.8 \pm 4.01$ & $<0.001$ \\
\hline Peak oxygen pulse (\%) & $79 \pm 16^{* *}$ & $69 \pm 15^{* *}$ & 0.002 \\
\hline \multicolumn{4}{|l|}{ Right ventricular contractility } \\
\hline Normal & 5 & 5 & \\
\hline Mildly reduced & 22 & 12 & \\
\hline Moderately reduced & 6 & 17 & \\
\hline Severely reduced & 1 & 0 & \\
\hline
\end{tabular}

Right ventricular dilatation

Normal 0

Mildly dilated

Moderately dilated

Severely dilated

Tricuspid regurgitation

$0,5-1 / 4$

21

$2 / 4$

$3 / 4$

$4 / 4$

$30 \quad 29$

23

Data are expressed as mean \pm standard deviation or as numbers of patients. 'Percentage of predicted values according to age and gender based on own control group.

Significantly different from $100 \%:{ }^{*} p<0.005 ;{ }^{* *} p<0.0001$.

most patients showed mildly reduced RV contractility and moderate RV dilation and all patients had some degree of tricuspid regurgitation. During the follow-up period, a decrease in RV contractility was reported in 10 patients, one patient showed an increase in RV

Table 3 Changes in exercise measures in \% per year by demographic variables

\begin{tabular}{llll}
\hline & $\begin{array}{l}\text { Peak oxygen } \\
\text { uptake }\end{array}$ & $\begin{array}{l}\text { Peak heart } \\
\text { rate }\end{array}$ & $\begin{array}{l}\text { Peak oxygen } \\
\text { pulse }\end{array}$ \\
\hline All $(n=34)$ & $-1.42 \pm 3.25^{*}$ & $-0.19 \pm 1.22$ & $-1.39 \pm 3.0^{*}$ \\
Gender & $-0.38 \pm 1.70$ & $0.15 \pm 0.70$ & $-0.50 \pm 1.94$ \\
Male $(n=22)$ & $-3.34 \pm 2.35$ & $-0.83 \pm 1.69$ & $-3.02 \pm 3.91^{*}$ \\
Female $(n=12)$ & & & \\
Age at first assessment & & $-0.30 \pm 1.20$ & $-1.92 \pm 3.64^{*}$ \\
$<16$ years $(n=18)$ & $-2.02 \pm 4.05^{*}$ & $-0.08 \pm 1.27$ & $-0.79 \pm 2.02$ \\
$\geq 16$ years $(n=16)$ & $-0.75 \pm 1.95$ & -1.02 \\
\hline
\end{tabular}

Data are expressed as mean \pm standard deviation.

* Significantly different from $0 \%(p<0.05)$. 
A

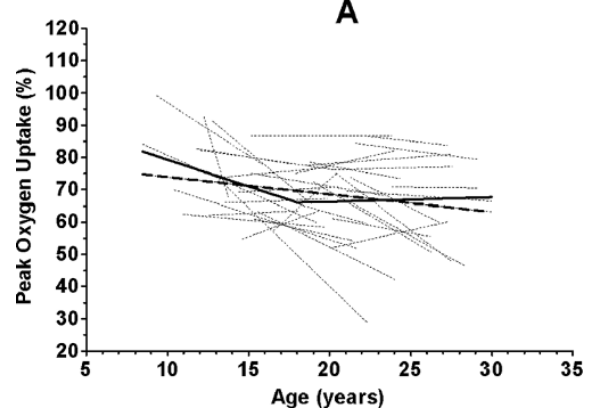

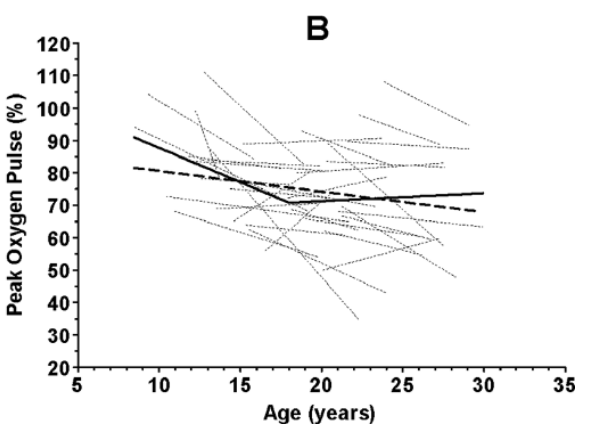

Figure 1 Change of percentage of predicted peak oxygen consumption (A) and peak oxygen pulse (B) as a function of age. The thicker line depicts the mean change in percentage of predicted values as calculated by segmental regression analysis in which slope and intercept are calculated before and after the age of 18 years. The thicker dotted line shows the mean percentage of predicted values in the population according to age calculated by linear regression analysis. Thin dotted lines show patient-specific linear regression lines.

dilatation, and 7 patients developed a worsening of the tricuspid valve incompetence.

During adulthood, the difference in RV contractility between the last and the first assessment was significantly correlated with the change in peak oxygen pulse $(\mathrm{r}=-0.5 ; \mathrm{p}=0.047)$.

\section{Discussion}

This study shows at first that patients with Senning operation for d-TGA have a lower exercise capacity, as expressed by peak $\mathrm{VO}_{2}$, peak oxygen pulse and peak heart rate, than what would be expected in a healthy population. Secondly, peak $\mathrm{VO}_{2}$ and peak oxygen pulse decrease faster with age than in healthy controls. This decline is most obvious during childhood and adolescence and in female patients. During early adulthood, peak $\mathrm{VO}_{2}$ and peak oxygen pulse show a similar evolution as in healthy peers. Thirdly, a decline in peak oxygen pulse in adults is associated with a decrease in RV contractility.

In previous research, several investigators have observed a lower exercise capacity in patients with atrial switch operation for d-TGA compared to a normal population and our results are in agreement with these observations $[2,6-8,11]$. The general decline of peak $\mathrm{VO}_{2}$ with age is well known $[3,6,12]$. Budts et al. suggested that this decrease in peak $\mathrm{VO}_{2}$ might be faster in adults with atrial switch repair since a strong negative relationship was found between cardiac index during submaximal exercise and age [7]. Our results showed a significantly faster decline of peak $\mathrm{VO}_{2}$ in our patients compared to the normal evolution, and our study thus provides some evidence for this reasoning. A possible explanation for a faster decline in exercise tolerance is the fact that these patients have a systemic RV, which is not designed to support the systemic circulation. The incapacity of the RV to increase stroke volume at higher exercise intensities was already shown to be probably causing reduced exercise capacity in patients with Senning repair [7]. An impaired atrio-ventricular transport which leads to a poor filling of the ventricle, and poor quality of the right ventricle and the valves might be one of the main determinants of an impaired stroke volume response to exercise [7]. It seems that the RV cannot keep performing against systemic pressures in the same manner as the left ventricle, causing the right ventricular function to decline with age, which at its turn contributes to a decline in exercise capacity. Our results show a weak but significant relationship between a decrease in oxygen pulse and a decrease in right ventricular contractility, supporting this reasoning. During growth, stroke volume increases when body mass increases for the same absolute rate of work. When stroke volume is estimated from the response of the oxygen pulse, our data suggest that children and adolescents with d-TGA cannot increase their stroke volume to the same extent as healthy peers.

Our results suggest that the decrease in peak $\mathrm{VO}_{2}$ and peak oxygen pulse occurred during childhood and adolescence, and was more stable during early adulthood. However, we believe that the small number of patients in the older age group resulted in a lack of power and therefore did not result in similar findings as Budts et al. [7]. Since the study population in the earlier mentioned investigation consisted of patients with Senning and Mustard operation, their different findings might also be triggered by possible differences between patients with Mustard and Senning operation. Reybrouck et al. also serially investigated the exercise tolerance, as assessed by the ventilatory threshold and the rate of increase in oxygen uptake, during medium term follow up (about 3 years in average) in children and adolescents with Mustard and Senning repair for d-TGA [3]. They reported that no significant change could be observed for exercise capacity. This is also in contradiction with our results, but we believe that the shorter follow-up period, smaller 
patient number, different population composition, younger age, other outcome measures and the fact that patients were only tested twice might account for these different findings.

The decrease in aerobic capacity in children and adolescents with atrial switch repair for d-TGA was cross sectionally studied by Fredriksen and colleagues and they also reported a significant decline in aerobic capacity with increasing age in young atrial switch patients [6]. They argued that chronotropic incompetence may be one of the reasons for this diminishing exercise capacity [6]. However, in our patient cohort, peak heart rate did not decline significantly and chronotropic incompetence was only present in a few patients. It is therefore unlikely that this might have influenced the overall change of the exercise capacity in our study group.

Furthermore, in patients with Senning operation, a reduced exercise capacity is often contributed to a lack of physical activity, which might be based on overprotection by the environment during childhood [13]. If patients with d-TGA have a low physical activity level during childhood and adolescence, this might counteract with the normal development of their exercise capacity and therefore explain the inability to increase the exercise capacity during growth to the same extent as healthy peers [13]. Moreover, it has recently been shown that adults with atrial switch repair for d-TGA show a reduced physical activity level in comparison with healthy counterparts, which is related to a lower exercise capacity [14]. Even though our data do not show a faster decline of the exercise capacity in young adults, we believe that on the longer term this sedentary lifestyle will probably contribute to the evolution of the exercise intolerance, making it decline faster than in a healthy and generally more active population.

Nowadays, the Senning operation is abandoned for routine palliation and replaced by the arterial switch operation [15]. However, Senning patients are still visiting specialized centres for adult congenital heart disease and often present with decreased systemic ventricular function and/or systemic atrioventricular valve regurgitation, which might deteriorate further with age and can lead to a larger decline of peak oxygen uptake and peak oxygen pulse than in a normal population. The care of patients with Senning operation for d-TGA who are growing older will therefore remain a huge challenge for the specialized cardiologists of our era in order to ensure the patients' health, well-being and quality of life.

\section{Study limitations}

A first limitation of our study is the low number of patients which does not allow that general conclusions are drawn. Secondly, our results can not be applied to patients older than 30 years of age. Thirdly, echocardiography has his limitations for the assessment of right ventricular function and size and our results remain to be confirmed by evaluations based on stronger cardiac imaging methods [16]. And finally, different exercise modalities were used for the children and the older patients. We are aware of the fact that results might be influenced by these differences; however we believe we take that into account by only working with percentages of predicted values in our analyses.

\section{Conclusions}

Patients with Senning operation for d-TGA have a lower exercise tolerance compared to a healthy population. Peak $\mathrm{VO}_{2}$ and peak oxygen pulse decline faster with age compared to healthy controls. This progressive decline is most obvious during childhood and adolescence and suggests the inability to increase stroke volume to the same extent as healthy peers during growth. Peak $\mathrm{VO}_{2}$ and peak oxygen pulse remain relatively stable during early adulthood. However when RV contractility decreases, a faster decline in peak oxygen pulse is observed.

\section{Abbreviations}

d-TGA: Complete transposition of the great arteries; peak $\mathrm{VO}_{2}$ : Peak oxygen uptake; CPET: Cardiopulmonary exercise tests; RV: Right ventricle/ventricular HR: Heart rate

\section{Competing interests}

The authors declare that they have no competing interests.

\section{Authors' contributions}

BR collected, cleaned and analyzed data, and she drafted and revised the manuscript. RT collected data and revised the manuscript. BW, GM and VL participated in the design of the study and revised the draft paper. All authors read and approved the final manuscript.

\section{Author details}

'Department of Rehabilitation Sciences, Research Center for Cardiovascular and Respiratory Rehabilitation, KULeuven, Tervuursevest 101, Leuven 3001, Belgium. ${ }^{2}$ Department of Cardiology, University Hospital Gasthuisberg, Herestraat 49, Leuven 3000, Belgium. ${ }^{3}$ Department of Cardiovascular Rehabilitation, University Hospital Gasthuisberg, Herestraat 49, Leuven 3000, Belgium. ${ }^{4}$ Department of Pediatric Cardiology, University Hospital Gasthuisberg, Herestraat 49, Leuven 3000, Belgium.

Received: 29 March 2012 Accepted: 11 October 2012 Published: 15 October 2012

\section{References}

1. Moons P, Sluysmans T, De Wolf D, Massin M, Suys B, Benatar A, Gewillig M: Congenital heart disease in 111225 births in Belgium: birth prevalence, treatment and survival in the 21st century. Acta Paediatr 2009, 98(3):472-477

2. Diller GP, Dimopoulos K, Okonko D, Li W, Babu-Narayan SV, Broberg CS, Johansson B, Bouzas B, Mullen MJ, Pooloe-Wilson BA, Francis DP, Gatzoulis MA: Exercise intolerance in adult congenital heart disease: comparative severity, correlates, and prognostic implication. Circulation 2005, 112(6):828-835.

3. Reybrouck T, Mertens L, Brown S, Eyskens B, Daenen W, Gewillig M: Longterm assessment and serial evaluation of cardiorespiratory exercise performance and cardiac function in patients with atrial switch operation for complete transposition. Cardiol Young 2001, 11(1):17-24. 
4. Fredriksen PM, Veldtman G, Hechter S, Therrien J, Chen A, Warsi MA Freeman M, Liu P, Siu S, Thaulow E, Webb G: Aerobic capacity in adults with various congenital heart diseases. Am J Cardiol 2001, 87(3):310-314.

5. Reybrouck T, Dumoulin M, van der Hauwaert LG: Cardiorespiratory exercise testing after venous switch operation in children with complete transposition of the great arteries. Am J Cardiol 1988, 61(10):861-865.

6. Fredriksen PM, Pettersen E, Thaulow E: Declining aerobic capacity of patients with arterial and atrial switch procedures. Pediatr Cardiol 2009, 30(2):166-171.

7. Budts W, Scheurwegs C, Stevens A, Moons P, Van Deyk K, Vanhees L: The future of adult patients after Mustard or Senning repair for transposition of the great arteries. Int J Cardiol 2006, 113(2):209-214.

8. Buys R, Cornelissen V, Van De Bruaene A, Stevens A, Coeckelberghs E, Onkelinx S, Thomaes T, Delecluse C, Budts W, Vanhees L: Measures of exercise capacity in adults with congenital heart disease. Int I Cardiol 2011, 153(1):26-30.

9. Giardini A, Hager A, Lammers AE, Derrick G, Müller J, Diller GP, Dimopoulos K, Odendaal D, Gargiulo G, Picchio FM, Gatzoulis MA: Ventilatory efficiency and aerobic capacity predict event-free survival in adults with atrial repair for complete transposition of the great arteries. J Am Coll Cardiol 2009, 53(17):1548-1555.

10. Reybrouck T, Weymans M, Stijns H, Knops J, van der Hauwaert L: Ventilatory anaerobic threshold in healthy children. Age and sex differences. Eur J Appl Physiol Occup Physiol 1985, 54(3):278-284.

11. Paul MH, Wessel HU: Exercise studies in patients with transposition of the great arteries after atrial repair operations (Mustard/Senning): a review. Pediatr Cardiol 1999, 20(1):49-55.

12. Reybrouck T, Rogers R, Weymans M, Dumoulin M, Vanhove M, Daenen W, Van Der Hauwaert L, Gewillig M: Serial cardiorespiratory exercise testing in patients with congenital heart disease. Eur J Pediatr 1995 154(10):801-806

13. Reybrouck T, Mertens L: Physical performance and physical activity in grown-up congenital heart disease. Eur J Cardiovasc Prev Rehabil 2005, 12(5):498-502.

14. Buys R, Van De Bruaene A, Budts W, Delecluse C, Vanhees L: A low physical activity level relates to reduced exercise capacity and decreased perceived physical functioning in adults with atrial switch operation for transposition of the great arteries. Acta Cardiol 2011, 67(1):49-57.

15. Van Praagh $R$, Jung WK: The arterial switch operation in transposition of the great arteries: anatomic indications and contraindications. Thorac Cardiovasc Surg 1991, 39(Suppl 2):138-150.

16. Lai WW, Gauvreau K, Rivera ES, Saleeb S, Powelle AJ, Geva T: Accuracy of guideline recommendations for two-dimensional quantification of the right ventricle by echocardiography. Int I Cardiovasc Imaging 2008, 24(7):691-698.

doi:10.1186/1471-2261-12-88

Cite this article as: Buys et al: Serial exercise testing in children, adolescents and young adults with Senning repair for transposition of the great arteries. BMC Cardiovascular Disorders 2012 12:88.

\section{Submit your next manuscript to BioMed Central and take full advantage of:}

- Convenient online submission

- Thorough peer review

- No space constraints or color figure charges

- Immediate publication on acceptance

- Inclusion in PubMed, CAS, Scopus and Google Scholar

- Research which is freely available for redistribution 